\title{
High versus low positive end-expiratory pressure during general anaesthesia for open abdominal surgery (PROVHILO trial): a multicentre randomised controlled trial
}

\author{
The PROVE Network Investigators* for the Clinical Trial Network of the European Society of Anaesthesiology
}

\section{Summary}

Background The role of positive end-expiratory pressure in mechanical ventilation during general anaesthesia for surgery remains uncertain. Levels of pressure higher than $0 \mathrm{~cm} \mathrm{H} \mathrm{H}_{2} \mathrm{O}$ might protect against postoperative pulmonary complications but could also cause intraoperative circulatory depression and lung injury from overdistension. We tested the hypothesis that a high level of positive end-expiratory pressure with recruitment manoeuvres protects against postoperative pulmonary complications in patients at risk of complications who are receiving mechanical ventilation with low tidal volumes during general anaesthesia for open abdominal surgery.

Methods In this randomised controlled trial at 30 centres in Europe and North and South America, we recruited 900 patients at risk for postoperative pulmonary complications who were planned for open abdominal surgery under general anaesthesia and ventilation at tidal volumes of $8 \mathrm{~mL} / \mathrm{kg}$. We randomly allocated patients to either a high level of positive end-expiratory pressure $\left(12 \mathrm{~cm} \mathrm{H}_{2} \mathrm{O}\right)$ with recruitment manoeuvres (higher PEEP group) or a low level of pressure ( $\leq 2 \mathrm{~cm} \mathrm{H}_{2} \mathrm{O}$ ) without recruitment manoeuvres (lower PEEP group). We used a centralised computergenerated randomisation system. Patients and outcome assessors were masked to the intervention. Primary endpoint was a composite of postoperative pulmonary complications by postoperative day 5 . Analysis was by intention-to-treat. The study is registered at Controlled-Trials.com, number ISRCTN70332574.

Findings From February, 2011, to January, 2013, 447 patients were randomly allocated to the higher PEEP group and 453 to the lower PEEP group. Six patients were excluded from the analysis, four because they withdrew consent and two for violation of inclusion criteria. Median levels of positive end-expiratory pressure were $12 \mathrm{~cm} \mathrm{H}_{2} \mathrm{O}$ (IQR 12-12) in the higher PEEP group and $2 \mathrm{~cm} \mathrm{H} \mathrm{H}_{2} \mathrm{O}(0-2)$ in the lower PEEP group. Postoperative pulmonary complications were reported in $174(40 \%)$ of 445 patients in the higher PEEP group versus $172(39 \%)$ of 449 patients in the lower PEEP group (relative risk 1.01; 95\% CI 0.86-1.20; $\mathrm{p}=0.86$ ). Compared with patients in the lower PEEP group, those in the higher PEEP group developed intraoperative hypotension and needed more vasoactive drugs.

Interpretation A strategy with a high level of positive end-expiratory pressure and recruitment manoeuvres during open abdominal surgery does not protect against postoperative pulmonary complications. An intraoperative protective ventilation strategy should include a low tidal volume and low positive end-expiratory pressure, without recruitment manoeuvres.

Funding Academic Medical Center (Amsterdam, Netherlands), European Society of Anaesthesiology.

\section{Introduction}

About 234 million major surgical procedures are undertaken worldwide every year. Of these interventions, around 2.6 million represent high-risk procedures, with 1.3 million patients developing complications that result in 315000 in-hospital deaths. ${ }^{1}$ Postoperative pulmonary complications are at least as frequent as cardiac complications during non-cardiac surgery ${ }^{2}$ and are associated with increased risk of in-hospital death, particularly after open abdominal surgery.,4 Mechanical ventilation might affect the incidence of postoperative pulmonary complications ${ }^{5}$ and, possibly, distal organ dysfunction. ${ }^{6}$ Different mechanisms have been proposed to account for the injurious effects of ventilation. Both hyperinflation and repetitive tidal recruitment of lung units can induce the release of proinflammatory mediators, leading to lung and distal organ injury.
Prevention of hyperinflation by use of low tidal volumes reduces mortality in patients with acute respiratory distress syndrome. ${ }^{8}$ Mortality can also be decreased in individuals with severe acute respiratory distress syndrome by avoiding repetitive tidal recruitment with high levels of positive end-expiratory pressure. ${ }^{9}$ Furthermore, use of low tidal volumes in patients without lung injury under general anaesthesia might also reduce the incidence of postoperative pulmonary complications. ${ }^{5}$ This hypothesis was proven in a single-centre ${ }^{10}$ and a national multicentre trial. ${ }^{11}$ However, in both studies, use of lower tidal volumes was combined with higher levels of positive end-expiratory pressure; thus, did beneficial effects come from prevention of hyperinflation or avoidance of repetitive tidal recruitment? Use of very low levels of positive end-expiratory pressure could lead to atelectasis with ventilation strategies that incorporate
Published Online June 1, 2014 http://dx.doi.org/10.1016/ S0140-6736(14)60416-5

See Online/Comment http://dx.doi.org/10.1016/ S0140-6736(14)60894-1 *PROVE (PROtective VEntilation) Network Investigators are listed in the appendix pp 1-3, and the Steering and Writing committees are listed at the end of the report Correspondence to: Dr Marcus J Schultz, Department of Intensive Care, Academic Medical Center at the University of Amsterdam, $1105 \mathrm{AZ}$ Amsterdam, Netherlands marcus.j.schultz@gmail.com

See Online for appendix 
lower tidal volumes. ${ }^{7,12}$ However, high levels of positive end-expiratory pressure might not only provoke complications such as intraoperative circulatory depression ${ }^{13}$ but also promote hyperinflation. ${ }^{14}$

We designed the PROtective Ventilation using HIgh versus LOw PEEP (PROVHILO) trial to test the hypothesis that a ventilation strategy with a high level of positive endexpiratory pressure plus recruitment manoeuvres during general anaesthesia for open abdominal surgery protects against postoperative pulmonary complications in patients at risk for complications.

\section{Methods}

\section{Study population}

We undertook a double-blind, parallel-group, randomised controlled trial at 30 hospitals in ten countries from Europe and North and South America. Participating hospitals are listed in the appendix (pp 2-3). We included patients aged 18 years or older who were scheduled for open abdominal surgery under general anaesthesia, but we restricted enrolment to individuals who had an intermediate or high risk of having postoperative pulmonary complications according to the ARISCAT score. ${ }^{4}$ We excluded patients who were planned for laparoscopic surgery, were pregnant (excluded by laboratory analysis), had a body-mass index higher than $40 \mathrm{~kg} / \mathrm{m}^{2}$, had severe cardiac or pulmonary comorbidities or another disorder that might have compromised safe trial procedure, or gave consent for another interventional study or declined to participate. Full inclusion and exclusion criteria are presented in the appendix (pp 8-9).

We obtained written informed consent from all participants before randomisation. The Institutional Review Boards of the Academic Medical Center (Amsterdam, Netherlands) and of all participating centres approved the study protocol and the statistical analysis plan. ${ }^{15}$ An independent Data Safety and Monitoring Board (appendix p 1) oversaw the trial, monitored patients' safety, and did interim analyses of masked data. Six participating centres were selected at random by the study monitor and the Steering committee (appendix p 1) and were visited by an independent observer to assess protocol adherence.

\section{Randomisation and masking}

We randomly allocated patients to receive intraoperative ventilation using either high levels of positive endexpiratory pressure $\left(12 \mathrm{~cm} \mathrm{H}_{2} \mathrm{O}\right)$ plus recruitment manoeuvres (higher PEEP group) or low levels of positive end-expiratory pressure $\left(\leq 2 \mathrm{~cm} \mathrm{H}_{2} \mathrm{O}\right)$ without recruitment manoeuvres (lower PEEP group). Local investigators did the random allocation after enrolment, using a secure, central, web-based randomisation system. The random sequence was computer-generated with a block size of four, stratified by centre. At every centre, at least two investigators obtained patients' data: one investigator was aware of the allocated intervention and obtained intraoperative data; the other remained unaware of the intraoperative interventions and assessed outcomes and scored postoperative pulmonary and extrapulmonary complications. The random allocation was also concealed from patients, research staff, the independent statistician, and the Data Safety and Monitoring Board.

\section{Outcomes}

The primary endpoint was a collapsed composite of postoperative pulmonary complications occurring in the first 5 days after surgery. These complications included hypoxaemia, severe hypoxaemia, bronchospasm, suspected pulmonary infection, pulmonary infiltrate, aspiration pneumonitis, development of acute respiratory distress syndrome, atelectasis, pleural effusion, pulmonary oedema caused by cardiac failure, and pneumothorax (appendix p 11).

A secondary and safety endpoint was intraoperative complications, which included: oxyhaemoglobin saturation $\left(\mathrm{SpO}_{2}\right)$ less than $90 \%$ and needing rescue; hypotension (ie, systolic arterial blood pressure $<90 \mathrm{~mm} \mathrm{Hg}$ for more than $3 \mathrm{~min}$ ); any need for vasoactive drugs; any new arrhythmias needing intervention; massive transfusion (ie, $>5$ units of packed-red-blood cells during $1 \mathrm{~h}$ ); and any surgical complication. Another secondary endpoint was postoperative extrapulmonary complications by postoperative day 5, which included: development of systemic inflammatory response syndrome; sepsis, severe sepsis, or septic shock; extrapulmonary infection; coma; acute myocardial infarction; acute renal failure; disseminated intravascular coagulation; hepatic failure; gastrointestinal bleeding; gastrointestinal failure; and impaired wound healing (appendix pp 12-13).

\section{Procedures}

The intraoperative ventilation protocol for both study groups is described in the appendix (p 10). Briefly, we ventilated patients during surgery using a volume-assist mode, with the option to switch to a pressure-support mode near the end of surgery. We set tidal volumes at $8 \mathrm{~mL} / \mathrm{kg}$ predicted bodyweight (PBW) and the fraction of inspired oxygen $\left(\mathrm{F}_{1} \mathrm{O}_{2}\right)$ at $0 \cdot 40$ or higher, to a target $\mathrm{SpO}_{2}$ of $92 \%$ or greater. We adjusted the respiratory rate to maintain end-tidal partial pressure of carbon dioxide $\left(\mathrm{FE}^{\prime} \mathrm{CO}_{2}\right)$ between $35 \mathrm{~mm} \mathrm{Hg}$ and $45 \mathrm{~mm} \mathrm{Hg}$, with an inspiration:expiration ratio of 1:2. Anaesthesiologists were allowed to change ventilator settings either on the surgeon's request or if concerns arose about the patient's safety. Safety concerns included: low systemic blood pressure unresponsive to intravenous fluids, vasoactive drugs, or both; new arrhythmias not responding to treatment; or need for a massive transfusion. Other aspects of general anaesthesia, fluid administration, and pain management were implemented according to usual routine.

In the higher PEEP group, recruitment manoeuvres consisted of incremental increases in tidal volume directly 
after induction of anaesthesia, after any disconnection from the ventilator, and just before tracheal extubation (appendix p 10). We designated a rescue strategy for patients in whom $\mathrm{SpO}_{2}$ measured by pulse oximetry fell to less than $90 \%$ without evidence of either airway problems, severe haemodynamic impairment, or ventilator malfunction (appendix p 10). The strategy included a stepwise increase of $\mathrm{F}_{1} \mathrm{O}_{2}$, a progressive rise in positive end-expiratory pressure, and recruitment manoeuvres. The rescue approach was implemented sequentially to return $\mathrm{SpO}_{2}$ to $92 \%$ or higher.

During surgery, local investigators who were aware of the random allocation recorded data on paper case report forms and, later, transferred this information to secure web-based electronic case report forms (OpenClinica, Boston, MA, USA). After surgery, different investigators who were unaware of the random allocation assessed patients daily, obtained clinical data, and scored presence of predefined outcomes and the need for admission to the intensive-care unit or readmission, until postoperative day 5 and shortly before hospital discharge. 90 days after surgery, we ascertained the number of hospital-free days (including admissions to other hospitals) and patients' vital status.

\section{Statistical analysis}

We calculated that a sample size of 900 patients would have $80 \%$ power to detect a difference in the incidence of postoperative pulmonary complications between the lower PEEP group (24\%) and the higher PEEP group $(16 \cdot 5 \%)^{2,4,10,16,17}$ The independent Data Safety and Monitoring Board undertook interim analyses after enrolment of 300 patients and 600 patients, according to the a-priori statistical analysis plan. The Board did not recommend trial discontinuation after either interim analysis; therefore, we continued with enrolment to 900 patients.

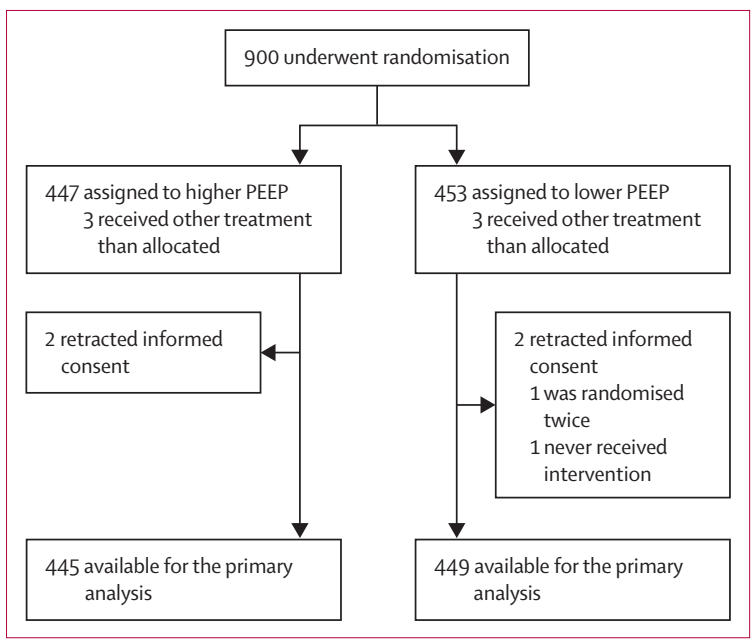

Figure 1: Trial profile

$\mathrm{PEEP}=$ positive end-expiratory pressure.
We analysed data by intention to treat. We compared postoperative variables with either Student's $t$ test or the Mann-Whitney $U$ test for continuous variables, depending on the characteristics of the variables, and we used the $\chi^{2}$ test for categorical variables. We compared both the composite primary outcome of incidence of postoperative pulmonary complications in the first 5 days after surgery and the secondary outcome of total occurrence of extrapulmonary complications

\begin{tabular}{|c|c|c|}
\hline & $\begin{array}{l}\text { Higher PEEP group } \\
(\mathrm{n}=445)\end{array}$ & $\begin{array}{l}\text { Lower PEEP group } \\
(n=449)\end{array}$ \\
\hline \multicolumn{3}{|l|}{ Demographic and clinical variables } \\
\hline Men & $259 / 445(58 \%)$ & $255 / 449(57 \%)$ \\
\hline Age (years) & $65(54-73)$ & $66(56-74)$ \\
\hline Body-mass index $\left(\mathrm{kg} / \mathrm{m}^{2}\right)$ & $25 \cdot 5(4 \cdot 2)$ & $25 \cdot 6(4 \cdot 4)$ \\
\hline Bodyweight $(\mathrm{kg})$ & $72 \cdot 5(14 \cdot 3)$ & $72 \cdot 7(14 \cdot 8)$ \\
\hline ARISCAT score* & $41(34-43)$ & $41(34-47)$ \\
\hline Intermediate (26-44) & $346 / 442(78 \%)$ & $331 / 447(74 \%)$ \\
\hline High (>44) & $98 / 442(22 \%)$ & $119 / 447(27 \%)$ \\
\hline \multicolumn{3}{|l|}{ Smoking status } \\
\hline Never & $245 / 445(55 \%)$ & $242 / 449(54 \%)$ \\
\hline Former & $111 / 445(25 \%)$ & $119 / 449(26 \%)$ \\
\hline Current & $91 / 445(20 \%)$ & $91 / 449(20 \%)$ \\
\hline \multicolumn{3}{|l|}{ Alcohol status (past 2 weeks) } \\
\hline None & $301 / 445(68 \%)$ & $307 / 447(69 \%)$ \\
\hline $0-2$ units & $130 / 445(29 \%)$ & $125 / 447(28 \%)$ \\
\hline$>2$ units & $16 / 445(4 \%)$ & $18 / 447(4 \%)$ \\
\hline \multicolumn{3}{|l|}{ ASA physical status classification system } \\
\hline 1 & $55 / 445(12 \%)$ & $54 / 448(12 \%)$ \\
\hline 2 & $246 / 445(55 \%)$ & $233 / 448(52 \%)$ \\
\hline 3 & $142 / 445(32 \%)$ & $156 / 448(35 \%)$ \\
\hline 4 & $3 / 445(1 \%)$ & $8 / 448(2 \%)$ \\
\hline 5 & $1 / 445(<1 \%)$ & 0 \\
\hline \multicolumn{3}{|l|}{ New York Heart Association classification } \\
\hline 1 & $347 / 435(80 \%)$ & $339 / 439(77 \%)$ \\
\hline ॥ & $87 / 435(20 \%)$ & $99 / 439(23 \%)$ \\
\hline III & $3 / 435(1 \%)$ & 4/439 (1\%) \\
\hline IV & 0 & 0 \\
\hline \multicolumn{3}{|l|}{ Functional status } \\
\hline Non-dependent & $427 / 445(96 \%)$ & $426 / 449(95 \%)$ \\
\hline Partly dependent & $18 / 445(4 \%)$ & $24 / 449(5 \%)$ \\
\hline Totally dependent & $2 / 445(<1 \%)$ & $2 / 449(<1 \%)$ \\
\hline History of active cancer & $268 / 441(61 \%)$ & $281 / 448(63 \%)$ \\
\hline History of chronic renal failure & $25 / 445(6 \%)$ & $22 / 449(5 \%)$ \\
\hline Chronic obstructive pulmonary disease & $37 / 445(8 \%)$ & $30 / 449(7 \%)$ \\
\hline With inhalation therapy $\dagger$ & $15 / 444(3 \%)$ & $15 / 448(3 \%)$ \\
\hline With systemic steroids & $8 / 444(2 \%)$ & $7 / 448(2 \%)$ \\
\hline Diabetes mellitus & $56 / 445(13 \%)$ & $79 / 449(18 \%)$ \\
\hline With oral medication & $38 / 54(70 \%)$ & $51 / 73(70 \%)$ \\
\hline With insulin & $16 / 54(30 \%)$ & $23 / 74(31 \%)$ \\
\hline Use of systemic steroids & $10 / 445(2 \%)$ & $8 / 448(2 \%)$ \\
\hline Use of statins & $82 / 445(18 \%)$ & $80 / 449(18 \%)$ \\
\hline \multirow[t]{2}{*}{ Preoperative transfusion } & $7 / 445(2 \%)$ & $10 / 448(2 \%)$ \\
\hline & \multicolumn{2}{|c|}{ (Table 1 continues on next page) } \\
\hline
\end{tabular}




\begin{tabular}{|c|c|c|}
\hline & $\begin{array}{l}\text { Higher PEEP group } \\
(\mathrm{n}=445)\end{array}$ & $\begin{array}{l}\text { Lower PEEP group } \\
(\mathrm{n}=449)\end{array}$ \\
\hline \multicolumn{3}{|l|}{ (Continued from previous page) } \\
\hline \multicolumn{3}{|l|}{ Preoperative tests } \\
\hline Haemoglobin (g/L) & $119(26)$ & $119(26)$ \\
\hline Creatinine $(\mu \mathrm{mol} / \mathrm{L})$ & $61(53-76)$ & $61(53-76)$ \\
\hline Urea (mmol/L) & $9 \cdot 3(5 \cdot 7-13)$ & $9 \cdot 6(5 \cdot 7-14)$ \\
\hline White blood cells $\left(\times 10^{9}\right.$ cells per $\left.\mathrm{L}\right)$ & $7(5 \cdot 7-8 \cdot 6)$ & $7(5 \cdot 7-8 \cdot 7)$ \\
\hline Preoperative oxyhaemoglobin saturation (\%)‡ & $97(96-98)$ & $97(96-98)$ \\
\hline Abnormalities on chest radiography & $23 / 329(7 \%)$ & $18 / 360(5 \%)$ \\
\hline \multicolumn{3}{|l|}{ Perioperative variables } \\
\hline Duration of surgery $(\mathrm{min}) \S$ & $200(140-300)$ & $190(140-262)$ \\
\hline \multicolumn{3}{|l|}{ Surgical procedure } \\
\hline Gastric & $42 / 445(9 \%)$ & $42 / 449(9 \%)$ \\
\hline Pancreatic & 60/445 (13\%) & 60/449 (13\%) \\
\hline Biliary & $15 / 445(3 \%)$ & $11 / 449(2 \%)$ \\
\hline Liver & $31 / 445(7 \%)$ & $31 / 449(7 \%)$ \\
\hline Colonic & $100 / 445(22 \%)$ & 98/449 (22\%) \\
\hline Rectal & 50/445 (11\%) & 48/449 (11\%) \\
\hline Bladder & $39 / 445(9 \%)$ & $47 / 449(10 \%)$ \\
\hline Kidney & $10 / 445(2 \%)$ & $12 / 449(3 \%)$ \\
\hline Vascular & $16 / 445(4 \%)$ & $18 / 449(4 \%)$ \\
\hline Other & $82 / 445(18 \%)$ & $82 / 449(18 \%)$ \\
\hline Antibiotic prophylaxis & 409/440 (93\%) & 411/449 (91\%) \\
\hline \multicolumn{3}{|l|}{ Type of anaesthesia } \\
\hline Total intravenous & $41 / 445(9 \%)$ & $41 / 449(9 \%)$ \\
\hline Mixed (volatile and intravenous) & 404/444 (91\%) & 408/448 (91\%) \\
\hline Epidural & 219/445 (49\%) & 226/449 (50\%) \\
\hline Thoracic & 173/218 (79\%) & $174 / 226(77 \%)$ \\
\hline Lumbar & 46/219 (21\%) & $52 / 226(23 \%)$ \\
\hline \multicolumn{3}{|c|}{$\begin{array}{l}\text { Data are mean (SD), median (IQR), or number/total number of patients (\%). ASA=American Society of Anesthesiolog } \\
\text { PEEP=positive end-expiratory pressure. "ARISCAT score measures risk of postoperative pulmonary complications. } \\
\text { tInhaled bronchodilators, steroids, or both. } ¥ \text { Measured by pulse oximetry. SDefined as the time between skin incision } \\
\text { and closure of the incision. }\end{array}$} \\
\hline
\end{tabular}

by postoperative day 5 with an unadjusted $\chi^{2}$ test, weighting every individual complication equally. We did not adjust the primary endpoint for baseline imbalance. In view of the two interim analyses, we regarded a two-sided $\alpha$ of 0.045 to be significant for the primary endpoint. We judged a $p$ value of less than 0.05 significant for other variables. Where appropriate, we expressed statistical uncertainty with 95\% CIs. We calculated Kaplan-Meier estimates of survival curves, and we used log-rank tests to compare survival distributions between study groups. We censored data used for Kaplan-Meier estimates when patients did not have a postoperative pulmonary complication during the study period, or when patients were lost to follow-up before the end of postoperative day 5 .

We did a post-hoc analysis on the primary endpoint, discarding the patients who developed hypoxaemia only from the composite endpoint of postoperative pulmonary complications, to allow comparison with previous studies.
Furthermore, we did an exploratory post-hoc per-protocol analysis, in which patients assigned to the higher PEEP group who did not receive high levels of positive endexpiratory pressure or recruitment manoeuvres (as indicated by the study protocol) were analysed as patients in the lower PEEP group. We did several other post-hoc assessments, including: a per-protocol analysis of intraoperative use of drugs (anaesthetics, neuromuscular blocking agents, and opioids); the net effect of the treatment group (higher PEEP) on the primary endpoint (postoperative pulmonary complications), controlling for centre; and a multiple logistic-regression analysis to identify baseline and intraoperative covariates associated with postoperative pulmonary complications.

We analysed data with R, version 2.3 (R Foundation for Statistical Computing, Vienna, Austria). This study is registered at Controlled-Trials.com, number ISRCTN70332574.

\section{Role of the funding source}

The European Society of Anaesthesiology and the Academic Medical Center (Amsterdam, Netherlands) financially supported and endorsed the trial. They had no role in study design, data collection, data analysis, data interpretation, or writing of the report. The Steering committee (appendix p 1) was responsible for accuracy and completeness of fidelity of the study to the protocol, data obtained, and data analyses. The Writing committee (appendix $\mathrm{p}$ 1) drafted the report without editorial assistance, and all Steering committee members made revisions and comments. JMB and SNTH had full access to all data in the study. SNTH, MJS, MGdA, and PP had final responsibility for the decision to submit for publication.

\section{Results}

From February, 2011, to January, 2013, we enrolled 900 patients from 30 centres in Europe and North and South America (figure 1). 447 individuals were randomly assigned to ventilation with a high level of positive endexpiratory pressure and recruitment manoeuvres (the higher PEEP group) and 453 participants were assigned to ventilation with a low level of positive end-expiratory pressure (the lower PEEP group). Randomisation of patients was balanced within centres (data not shown). Four people retracted their informed consent after randomisation, one patient did not receive treatment, and another individual was randomised twice, and these six people were excluded from the intention-to-treat analysis. Another six patients received treatment different to that allocated but were included in the intention-to-treat analysis. Therefore, data for the primary endpoint could be analysed for 445 patients in the higher PEEP group and 449 individuals in the lower PEEP group. However, 14 patients were lost to follow-up and, thus, data could not be obtained for the primary endpoint: one individual in the higher PEEP group 
transferred to another hospital; one person in the higher PEEP group was admitted to intensive care; follow-up was mistakenly not done because of communication errors for five patients in the higher PEEP group and three in the lower PEEP group; and one individual in the higher PEEP group and three in the lower PEEP group had missing follow-up data for unknown reasons.

Baseline characteristics did not differ between treatment groups (table 1). Just under two-thirds of surgical procedures were for cancer. During surgery, median tidal volumes were similar between study groups (table 2) and they remained within the target range throughout intraoperative mechanical ventilation. Median positive end-expiratory pressure levels were 12 (IQR 12-12) cm $\mathrm{H}_{2} \mathrm{O}$ in the higher PEEP group and $2(0-2) \mathrm{cm} \mathrm{H}_{2} \mathrm{O}$ in the lower PEEP group. 438 (99\%) patients received recruitment manoeuvres after intubation in the higher PEEP group compared with six (1\%) patients in the lower PEEP group (table 2); 378 (85\%) patients in the higher PEEP group and three $(1 \%)$ in the lower PEEP group received recruitment manoeuvres before extubation (appendix p 14). Peak pressure, dynamic respiratory compliance, and $\mathrm{SpO}_{2}$ were significantly higher in the higher PEEP group than in the lower PEEP group (table 2). 11 (2\%) patients allocated to the higher PEEP group needed rescue for desaturation versus $34(8 \%)$ in the lower PEEP group (relative risk 0.34 , 95\% CI 0.18-0.67; p=0 0008; table 3, appendix p 15). In 34 patients assigned to the higher PEEP group, positive end-expiratory pressure was decreased at the request of the surgeon $(n=5)$ or the attending anaesthesiologist $(n=3)$, because of hypotension $(n=14)$ or massive surgical bleeding $(n=10)$, or for other reasons $(n=2)$.

Haemodynamic compromise happened more frequently during the high positive end-expiratory pressure strategy (relative risk $1 \cdot 29$, 95\% CI 1.10-1.51; $\mathrm{p}=0 \cdot 0016$; table 3 ). Patients assigned to the higher PEEP group had a greater need for vasopressors $(1 \cdot 20$, 1.07-1.35; $\mathrm{p}=0 \cdot 0016)$ and received more fluids than did individuals allocated to the lower PEEP group (table 2). The duration of surgery, administration of anaesthesia, use of epidural anaesthesia, intraoperative blood loss, transfusion of blood products, arrhythmias, surgical complications, or urine output did not differ between groups (tables 1-3, appendix pp 16-17).

Postoperative pulmonary complications within the first 5 days after surgery were recorded in 174 (40\%) of 437 patients in the higher PEEP group versus $172(39 \%)$ of 443 individuals in the lower PEEP group (relative risk 1.01, 95\% CI 0.85-1.20; $\mathrm{p}=0 \cdot 84$; table 3 , figure 2 ). The need for continued or new postoperative mechanical ventilation did not differ between groups, with 18 (4\%) patients needing ventilation after surgery in the higher PEEP group versus $24(5 \%)$ in the lower PEEP group $(0 \cdot 77$, $0 \cdot 42-1.40 ; \mathrm{p}=0 \cdot 74)$. Hypoxaemia was reported in just under a quarter of patients; discarding this complication from the composite primary endpoint of postoperative

\begin{tabular}{|c|c|c|c|}
\hline & $\begin{array}{l}\text { Higher PEEP group } \\
(n=445)\end{array}$ & $\begin{array}{l}\text { Lower PEEP group } \\
(\mathrm{n}=449)\end{array}$ & $p$ \\
\hline Tidal volumes (mL) & $500(450-560)$ & $500(450-550)$ & .. \\
\hline PBW (mL/kg) & $7 \cdot 2(1 \cdot 5)$ & $7 \cdot 1(1 \cdot 2)$ & .. \\
\hline After $1 \mathrm{~h}$ & $7 \cdot 11(1.32)$ & $7.09(1.23)$ & .. \\
\hline Directly before extubation & $6.96(1.50)$ & $7.07(1.23)$ & .. \\
\hline $\operatorname{PEEP}\left(\mathrm{cm} \mathrm{H}_{2} \mathrm{O}\right)$ & $12(12-12)$ & $2(0-2)$ & .. \\
\hline After $1 \mathrm{~h}$ & $12(12-12)$ & $2(0-2)$ & \\
\hline Directly before extubation & $12(12-12)$ & $2(0-2)$ & \\
\hline Peak pressure $\left(\mathrm{mL} / \mathrm{cm} \mathrm{H}_{2} \mathrm{O}\right)$ & $23(3 \cdot 7)$ & $17(4 \cdot 1)$ & .. \\
\hline After $1 \mathrm{~h}$ & $23 \cdot 1(4 \cdot 1)$ & $16.8(4 \cdot 4)$ & .. \\
\hline Directly before extubation & $22 \cdot 7(4 \cdot 2)$ & $16 \cdot 7(4 \cdot 1)$ & .. \\
\hline Calculated $\mathrm{Cdyn}\left(\mathrm{mL} / \mathrm{cm} \mathrm{H}_{2} \mathrm{O}\right)$ & $44(35-54)$ & $34(27-41)$ & $<0.0001$ \\
\hline Begin* & $45(36-57)$ & $33(27-43)$ & $<0.0001$ \\
\hline End $\dagger$ & $44(36-54)$ & $35(27-42)$ & $<0.0001$ \\
\hline Respiratory rate (breaths/min) & $11(2 \cdot 1)$ & $11(1 \cdot 9)$ & 0.13 \\
\hline Minute ventilation (mL/min) & $5681(1267)$ & 5545 (1162) & 0.10 \\
\hline $\mathrm{F}_{1} \mathrm{O}_{2}(\%) \ddagger$ & $40(40-49)$ & $41(40-50)$ & 0.06 \\
\hline$<40$ & $222 / 445(50 \%)$ & 202/449 (45\%) & 0.14 \\
\hline $40-60$ & 190/445 (43\%) & 206/449 (46\%) & 0.34 \\
\hline $60-80$ & $4(18 / 445)$ & $5(22 / 449)$ & 0.54 \\
\hline$>80$ & $3(15 / 445)$ & $4(19 / 449)$ & 0.50 \\
\hline $\mathrm{SpO}_{2}(\%) \Omega$ & $99(98 \cdot 5-100)$ & $99(98-99.8)$ & $<0.0001$ \\
\hline $\mathrm{FE}^{\prime} \mathrm{CO}_{2}(\mathrm{~mm} \mathrm{Hg})$ & $35 \cdot 2(3 \cdot 7)$ & $34 \cdot 5(3 \cdot 4)$ & 0.0007 \\
\hline Blood pressure $(\mathrm{mm} \mathrm{Hg}) \ddagger$ & $77 \cdot 8(9 \cdot 8)$ & $77 \cdot 9(10)$ & 0.28 \\
\hline$>70$ & $61(270 / 445)$ & $60(269 / 449)$ & 0.82 \\
\hline $60-70$ & $31(137 / 445)$ & $30(134 / 449)$ & 0.76 \\
\hline$<60$ & $9(38 / 445)$ & $10(46 / 449)$ & 0.38 \\
\hline Heart rate (bpm) & $70 \cdot 7(12 \cdot 7)$ & $68.8(10.9)$ & 0.0121 \\
\hline \multicolumn{4}{|l|}{ Recruitment manoeuvre done } \\
\hline After intubation & $438 / 442(99 \%)$ & 6/452 (1\%) & .. \\
\hline Before extubation & $378 / 444(85 \%)$ & $3 / 429(1 \%)$ & .. \\
\hline Crystalloids given (mL) & $2200(1500-3100)$ & $2000(1400-3000)$ & 0.0229 \\
\hline Colloids given (mL) & $500(0-1000)$ & $500(0-1000)$ & 0.30 \\
\hline \multicolumn{4}{|l|}{ Total fluids (crystalloids and colloids) } \\
\hline$<1000 \mathrm{~mL}$ & $22 / 436(5 \%)$ & $41 / 435(9 \%)$ & 0.0126 \\
\hline $1000-3000 \mathrm{~mL}$ & $236 / 436(54 \%)$ & 245/435 (56\%) & 0.52 \\
\hline 3000-5000 mL & $131 / 436(30 \%)$ & 111/435 (26\%) & 0.14 \\
\hline$>5000 \mathrm{~mL}$ & $47 / 436(11 \%)$ & $38 / 435(9 \%)$ & 0.31 \\
\hline Urine output (mL) & $300(187-560)$ & $340(200-600)$ & 0.32 \\
\hline Transfusion of packed-red-blood cells & $62 / 443(14 \%)$ & $78 / 449(17 \%)$ & 0.24 \\
\hline Transfusion of fresh-frozen plasma & $21 / 420(5 \%)$ & $24 / 449(5 \%)$ & 0.82 \\
\hline Transfusion of platelets & $3 / 429(1 \%)$ & $10 / 449(2 \%)$ & 0.056 \\
\hline Blood loss (mL) & $500(200-1000)$ & $400(200-800)$ & 0.38 \\
\hline Massive transfusion needed $\mathbb{T}$ & $12 / 444(3 \%)$ & $5 / 445(1 \%)$ & 0.09 \\
\hline Temperature at end of surgery $\left({ }^{\circ} \mathrm{C}\right)$ & $36(0 \cdot 6)$ & $36(0.6)$ & 0.58 \\
\hline Perforation of organ & 4/444 (1\%) & 4/444 (1\%) & $>0.99$ \\
\hline \multicolumn{4}{|c|}{ 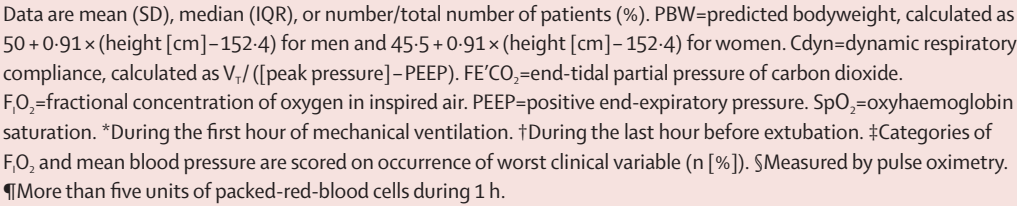 } \\
\hline
\end{tabular}


pulmonary complications did not alter the initial finding, and no difference was seen between groups (table 3 ). No heterogeneity across centres was noted for postoperative pulmonary complications (appendix p 5).

\begin{tabular}{|c|c|c|c|c|}
\hline & $\begin{array}{l}\text { Higher PEEP } \\
\text { group }(n=445)\end{array}$ & $\begin{array}{l}\text { Lower PEEP } \\
\text { group }(n=449)\end{array}$ & $\begin{array}{l}\text { Relative risk } \\
(95 \% \mathrm{Cl})\end{array}$ & $\mathrm{p}$ \\
\hline \multicolumn{5}{|l|}{ Postoperative pulmonary complications } \\
\hline Total $^{*}$ & $174 / 437(40 \%)$ & $172 / 443(39 \%)$ & $1.01(0.85-1.20)$ & 0.84 \\
\hline Total (excluding hypoxaemia) & $142 / 437(32 \%)$ & $149 / 443(34 \%)$ & $0.96(0.78-1.17)$ & 0.66 \\
\hline Hypoxaemia & $105 / 437(24 \%)$ & $95 / 443(21 \%)$ & $1.08(0.92-1.25)$ & 0.36 \\
\hline Severe hypoxaemia & $29 / 437(7 \%)$ & $34 / 443(8 \%)$ & $0.92(0.70-1.21)$ & 0.55 \\
\hline Bronchospasm & $18 / 437(4 \%)$ & $18 / 443(4 \%)$ & $1.01(0.72-1.41)$ & 0.97 \\
\hline Suspected pulmonary infection & $68 / 437(16 \%)$ & $75 / 443(17 \%)$ & $0.95(0.79-1.14)$ & 0.58 \\
\hline Pulmonary infiltrate & $35 / 437(8 \%)$ & $32 / 443(7 \%)$ & $1.06(0.83-1 \cdot 34)$ & 0.66 \\
\hline Aspiration pneumonitis & $1 / 437(<1 \%)$ & $4 / 443(1 \%)$ & $0.40(0.07-2 \cdot 32)$ & 0.18 \\
\hline Acute respiratory distress syndrome & $5 / 437(1 \%)$ & $8 / 443(2 \%)$ & $0.77(0.39-1 \cdot 54)$ & 0.41 \\
\hline Atelectasis & $53 / 437(12 \%)$ & $55 / 443(12 \%)$ & $0.99(0.80-1.21)$ & 0.90 \\
\hline Pleural effusion & $90 / 437(21 \%)$ & $92 / 443(21 \%)$ & $0.99(0.84-1.17)$ & 0.95 \\
\hline $\begin{array}{l}\text { Pulmonary oedema caused by cardiac } \\
\text { failure }\end{array}$ & $19 / 437(4 \%)$ & $20 / 443(5 \%)$ & $0.98(0.71-1 \cdot 36)$ & 0.90 \\
\hline Pneumothorax & $15 / 437(3 \%)$ & $12 / 443(3 \%)$ & $1.12(0.80-1.58)$ & 0.53 \\
\hline \multicolumn{5}{|c|}{ Postoperative extrapulmonary complications } \\
\hline Total extrapulmonary complications & $244 / 445(55 \%)$ & $242 / 449(54 \%)$ & $1.02(0.90-1.15)$ & 0.78 \\
\hline $\begin{array}{l}\text { Systemic inflammatory response } \\
\text { syndrome }\end{array}$ & $62 / 437(14 \%)$ & $64 / 443(14 \%)$ & $0.97(0.70-1.35)$ & 0.91 \\
\hline Sepsis & $18 / 437(4 \%)$ & $18 / 443(4 \%)$ & $1.01(0.53-1.91)$ & 0.96 \\
\hline Severe sepsis & $5 / 437(1 \%)$ & $4 / 443(1 \%)$ & $1.26(0.34-4.67)$ & 0.72 \\
\hline Septic shock & $3 / 437(1 \%)$ & $3 / 443(1 \%)$ & $1.01(0.20-4.97)$ & 0.98 \\
\hline Extrapulmonary infections & $34 / 437(8 \%)$ & $31 / 443(7 \%)$ & $1.11(0.69-1.77)$ & 0.66 \\
\hline Coma & $1 / 437(<1 \%)$ & $1 / 443(<1 \%)$ & $1.01(0.06-16)$ & 0.49 \\
\hline Acute myocardial infarction & 6/437 (1\%) & $5 / 443(1 \%)$ & $1.21(0.37-3.94)$ & 0.74 \\
\hline Acute renal failure (RIFLE criteria) ${ }^{\dagger}$ & & & & 0.60 \\
\hline None & $342 / 391(87 \%)$ & 341/397 (86\%) & $1.02(0.96-1.08)$ & 0.52 \\
\hline Risk $\neq$ & $34 / 391(9 \%)$ & $33 / 397(8 \%)$ & $1.05(0.66-1.65)$ & 0.85 \\
\hline Injurys & $8 / 391(2 \%)$ & $14 / 397(4 \%)$ & $0.58(0.25-1.37)$ & 0.21 \\
\hline Failureף & 7/391 (2\%) & 9/397 (2\%) & $0.79(0.30-2.10)$ & 0.64 \\
\hline Loss $\|$ & $1 / 391(<1 \%)$ & 0 & .. &. \\
\hline $\begin{array}{l}\text { Disseminated intravascular } \\
\text { coagulation }\end{array}$ & $1 / 437(<1 \%)$ & 0 & $0.14(0.02-1 \cdot 17)$ & 0.16 \\
\hline Hepatic failure & $32 / 445(7 \%)$ & $34 / 449(8 \%)$ & $0.95(0.60-1.52)$ & 0.84 \\
\hline Gastrointestinal bleeding & 3/ $437(1 \%)$ & $6 / 443(1 \%)$ & $0.51(0.13-2.03)$ & 0.32 \\
\hline Gastrointestinal failure $\dagger^{* *}$ & & & & 0.94 \\
\hline 0 & 197/394 (50\%) & 193/399 (48\%) & $1.03(0.89-1.20)$ & 0.79 \\
\hline 1 & $162 / 394(41 \%)$ & $168 / 399(42 \%)$ & $0.98(0.82-1.18)$ & 0.86 \\
\hline 2 & $33 / 394(8 \%)$ & 35/399 (9\%) & $0.96(0.61-1.51)$ & 0.85 \\
\hline 3 & 2/394 (1\%) & 3/399 (1\%) & $0.68(0.11-4.03)$ & 0.66 \\
\hline 4 & 0 & 0 & .. & \\
\hline \multicolumn{5}{|l|}{ Intraoperative complications } \\
\hline Rescue strategy for desaturation & $11 / 442(2 \%)$ & $34 / 445(8 \%)$ & $0.34(0.18-0.67)$ & 0.0008 \\
\hline Hypotensiont† & $205 / 441(46 \%)$ & $162 / 449(36 \%)$ & $1 \cdot 29(1 \cdot 10-1 \cdot 51)$ & 0.0016 \\
\hline Vasoactive drugs needed & $274 / 444(62 \%)$ & $228 / 445(51 \%)$ & $1 \cdot 20(1 \cdot 07-1 \cdot 35)$ & 0.0016 \\
\hline New arrhythmias needing & $12 / 442(3 \%)$ & $5 / 445(1 \%)$ & $2.38(0.84-6.70)$ & 0.09 \\
\hline
\end{tabular}

(Table 3 continues on next page)
In the higher PEEP group, 244 (55\%) patients developed extrapulmonary complications versus 242 (54\%) in the lower PEEP group (relative risk 1.02, 95\% CI 0 - 90-1.15; $\mathrm{p}=0 \cdot 78$; table 3 , appendix $\mathrm{p} 7$ ). In both treatment groups, gastrointestinal failure was the most common extrapulmonary complication, followed by systemic inflammatory response syndrome and acute renal failure (table 3). Admission to the intensive-care unit, the number of hospital-free days at postoperative day 90 , and in-hospital mortality did not differ between groups (table 3).

The results of per-protocol analyses did not differ from those of the intention-to-treat analyses (appendix p 18). Findings of further post-hoc analyses are presented in the appendix (pp 6, 17, and 19).

\section{Discussion}

The findings of our randomised trial show that, in patients having open abdominal surgery under general anaesthesia and with mechanical ventilation, the incidence of postoperative pulmonary complications is comparable in the first 5 days after surgery between patients receiving a high level of positive end-expiratory pressure and recruitment manoeuvres and those receiving a low level of positive end-expiratory pressure only. PROVHILO is the first study to incorporate identical low tidal volumes into both treatment groups, enabling the effects of high levels of positive endexpiratory pressure to be isolated from the known outcomes of tidal volume size (panel).

Our composite endpoint of postoperative pulmonary complications included hypoxaemia, which was the most common complication. Restricting our analysis to more severe postoperative pulmonary complications did not change the study results, suggesting that the level of positive end-expiratory pressure does not alter the risk of more severe pulmonary complications. The incidence of postoperative pulmonary complications in our trial was substantially higher than in previous studies, ${ }^{2,4,10,16,17}$ which might have been attributable to inclusion of patients at much higher risk of developing postoperative pulmonary complications compared with individuals in previous studies. Because the recorded incidence of complications was so high, our trial had sufficient statistical power to detect a difference in the frequency of postoperative pulmonary complications of $7 \cdot 5 \%$. We aimed to reduce the risk of bias by using centralised randomisation and by masking outcome assessors to the study group assignment. We used a relevant composite outcome at a meaningful interval in this surgical population. Moreover, we published the statistical analysis plan before we unmasked the study group assignments. ${ }^{15}$

The chosen level of positive end-expiratory pressure used in the higher PEEP group is supported by scientific literature. ${ }^{19,20}$ Previous studies tested levels of positive end-expiratory pressure of $10 \mathrm{~cm} \mathrm{H}_{2} \mathrm{O}$ during intraoperative ventilation, ${ }^{21-23}$ but atelectasis persisted during 
anaesthesia in some patients, particularly when high amounts of $\mathrm{F}_{1} \mathrm{O}_{2}$ were used. ${ }^{23}$ Notably, atelectasis might also persist in the first days after surgery, particularly after abdominal surgery. ${ }^{24}$ We chose a positive endexpiratory pressure of $12 \mathrm{~cm} \mathrm{H}_{2} \mathrm{O}$ to maximise lung opening throughout mechanical ventilation, irrespective of $\mathrm{F}_{1} \mathrm{O}_{2}$. The higher PEEP strategy resulted in improved dynamic compliance of the respiratory system compared with that noted in the lower PEEP group, suggesting augmented alveolar recruitment.

The results of PROVHILO expand our understanding of the findings of two trials in similar populations of patients,,$^{10,11}$ in which a conventional ventilation strategy with high tidal volumes of $9.5 \mathrm{~mL} / \mathrm{kg} \mathrm{PBW}^{10}$ and $11.1 \mathrm{~mL} / \mathrm{kg} \mathrm{PBW}^{11}$ and no positive end-expiratory pressure was compared with a protective strategy using low tidal volumes of $7.7 \mathrm{~mL} / \mathrm{kg} \mathrm{PBW}^{10}$ and $6.4 \mathrm{~mL} / \mathrm{kg} \mathrm{PBW}^{11}$ and high levels of positive end-expiratory pressure of $10 \mathrm{~cm}$ $\mathrm{H}_{2} \mathrm{O}^{10}$ and $6 \mathrm{~cm} \mathrm{H}_{2} \mathrm{O} .{ }^{11}$ The benefit of protective ventilation reported in those trials might have come from the high levels of positive end-expiratory pressure. ${ }^{25}$ However, the design of the trials ${ }^{10,11}$ does not enable us to identify whether low tidal volumes, high levels of positive endexpiratory pressure, or both, caused the beneficial effects (panel). The results of our study, therefore, challenge the hypothesis that high positive end-expiratory pressure accounts for the beneficial effects of protective ventilation. However, the two trials ${ }^{10,11}$ are not completely comparable with our study, because the levels of high positive endexpiratory pressure used were about $4-6 \mathrm{~cm} \mathrm{H}_{2} \mathrm{O}$ lower than those we administered.

Perhaps, in our trial, the high level of positive endexpiratory pressure stabilised the lungs and protected against lung injury from tidal recruitment, but the adverse effects we recorded might have counteracted these possible beneficial effects. Peak airway pressures were increased in patients assigned to the higher PEEP group, possibly causing hyperinflation in non-dependent lung zones. Furthermore, high positive end-expiratory pressure further impaired haemodynamics. Thus, our findings suggest that levels of positive end-expiratory pressure higher than recommended in previous trials,,$^{10,11}$ although improving the elastic properties of the respiratory system, do not enhance lung protection in general anaesthesia.

Several drugs used for general anaesthesia induce peripheral vascular smooth muscle relaxation, decrease the arterial pressure, and, even, impair cardiac contractility. ${ }^{26,27}$ Furthermore, epidural anaesthesia, which is used frequently (in up to $50 \%$ of cases) in combination with general anaesthesia during open abdominal surgery, might contribute to reduce the peripheral vascular smooth muscle tonus and promote peripheral blood pooling. ${ }^{28}$ However, neither administration of drugs for general anaesthesia nor use of epidural anaesthesia differed between study groups. Thus, the increased incidence of intraoperative haemodynamic adverse events noted in the higher PEEP group, particularly arterial

\begin{tabular}{|c|c|c|c|c|}
\hline & $\begin{array}{l}\text { Higher PEEP } \\
\text { group }(n=445)\end{array}$ & $\begin{array}{l}\text { Lower PEEP } \\
\text { group }(n=449)\end{array}$ & $\begin{array}{l}\text { Relative risk } \\
(95 \% \mathrm{Cl})\end{array}$ & $\mathrm{p}$ \\
\hline \multicolumn{5}{|l|}{ (Continued from previous page) } \\
\hline \multicolumn{5}{|l|}{ Follow-up } \\
\hline Impaired wound healing $\neq \ddagger$ & $71 / 444(16 \%)$ & $58 / 446(13 \%)$ & $1.23(0.89-1.70)$ & $0 \cdot 21$ \\
\hline $\begin{array}{l}\text { Need for new or continued } \\
\text { mechanical ventilation }\end{array}$ & $18 / 437(4 \%)$ & $24 / 443(5 \%)$ & $0.77(0 \cdot 42-1 \cdot 40)$ & $0 \cdot 74$ \\
\hline Admission to intensive-care unit & $106 / 442(24 \%)$ & $104 / 452(23 \%)$ & $1.03(0.81-1 \cdot 32)$ & 0.79 \\
\hline Length of hospital stay (days) & $10(7-14)$ & $10(7-14)$ & .. & 0.24 \\
\hline Hospital-free days, at day 90 & $79(71-83)$ & $79(70-82)$ & .. & $0 \cdot 33$ \\
\hline Mortality by day 5 & $2 / 443(<1 \%)$ & $1 / 448(<1 \%)$ & $2.02(0.18-22)$ & 0.56 \\
\hline In-hospital mortality & $7 / 438(2 \%)$ & $7 / 442(2 \%)$ & $1.01(0.36-2.85)$ & 0.99 \\
\hline \multicolumn{5}{|c|}{ 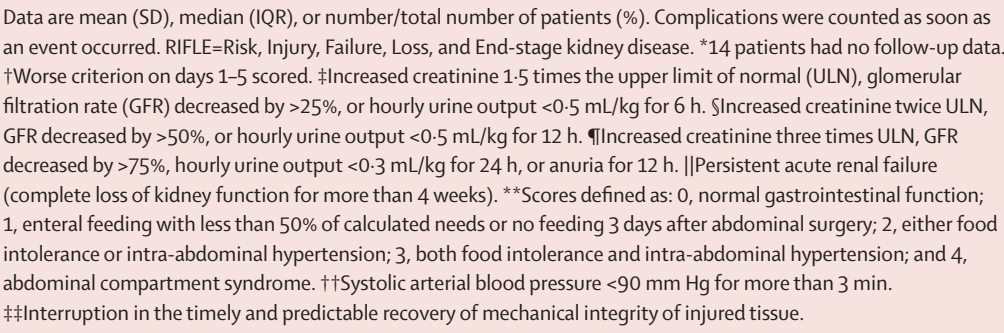 } \\
\hline
\end{tabular}

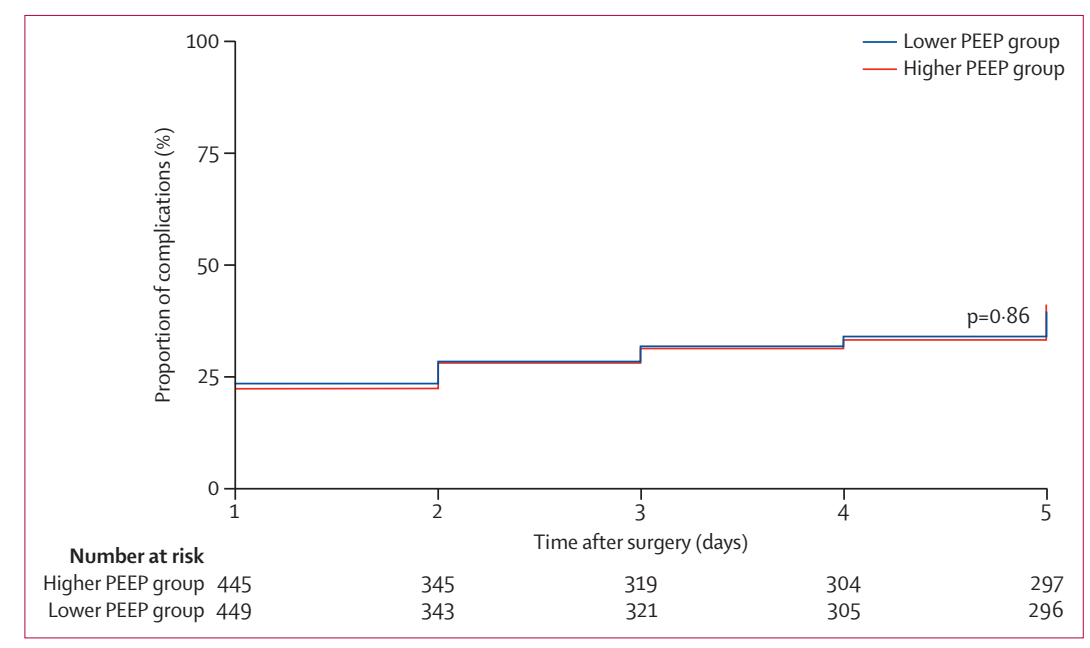

Figure 2: Kaplan-Meier curve showing the probability of postoperative pulmonary complications by postoperative day 5

$\mathrm{PEEP}=$ positive end-expiratory pressure.

hypotension, might have been associated with a reduction of venous return attributable to increased intrathoracic pressure with higher positive end-expiratory pressure and recruitment manoeuvres. Even though those events were scarce and responded to increased intravascular volume expansion and use of vasoactive drugs, they might be lifethreatening in patients with ischaemic cardiac disease. ${ }^{29}$

In our study, we did not include patients having laparoscopic surgery or those who were morbidly obese-groups of patients who might have benefited, in particular, from high levels of intraoperative positive end-expiratory pressure. Furthermore, we recommended, 
Panel: Research in context

\section{Systematic review}

We have previously undertaken two meta-analyses on intraoperative ventilation, ${ }^{5,18}$ for which we searched Medline, the Cumulative Index to Nursing and Allied Health Literature (CINAHL), Web of Science, and the Cochrane Central Register of Controlled Trials (CENTRAL) between 1966 and 2013, with the MeSH keywords "protective ventilation" and "lower tidal volumes". We identified 2122 studies on mechanical ventilation and selected those that both included patients solely with uninjured lungs and evaluated two ventilation strategies-one with low tidal volumes (protective ventilation) and one with high tidal volumes (conventional ventilation). The primary endpoint of the meta-analyses was development of lung injury. The findings showed that protective ventilation in patients without lung injury was associated with reduced pulmonary complications compared with conventional ventilation, both in the intensive-care unit and during general anaesthesia for surgery. Two trials of intraoperative ventilation confirmed these findings. ${ }^{10,11} \mathrm{~A}$ combined intervention was compared in these two trials and in most of the studies included in the meta-analyses: use of low tidal volumes and high levels of positive end-expiratory pressure with recruitment manoeuvres. Whether high levels of positive end-expiratory pressure with recruitment manoeuvres add to the beneficial effect of low tidal volumes is uncertain. Furthermore, use of high levels of positive end-expiratory pressure with recruitment manoeuvres could induce haemodynamic compromise. Thus, we investigated whether a high level of positive end-expiratory pressure with recruitment manoeuvres versus a strategy of low positive end-expiratory pressure could protect against postoperative pulmonary complications in patients undergoing open abdominal surgery under general anaesthesia and with mechanical ventilation at low tidal volumes.

\section{Interpretation}

As far as we are aware, our study is the largest multicentre, international, randomised controlled trial to date of mechanical ventilation during general anaesthesia for open abdominal surgery. A strategy using a high level of positive end-expiratory pressure and recruitment manoeuvres did not reduce the incidence of postoperative pulmonary complications when compared with a strategy using low levels of positive end-expiratory pressure without recruitment manoeuvres, yet it increased intraoperative circulatory impairment. Our findings might change current practice of mechanical ventilation during general anaesthesia for open abdominal surgery. A protective mechanical ventilation strategy with a low tidal volume does not gain from high positive end-expiratory pressure with recruitment manoeuvres. If intraoperative desaturation happens, we advise to increase the inspired oxygen fraction before raising positive end-expiratory pressure and undertaking lung recruitment manoeuvres.

but did not reinforce, use of international guidelines and standards for intraoperative and postoperative fluid administration, use of inotropes and vasopressors, and use or reversal of neuromuscular blocking agents. Our study was pragmatic in its design, rather than being controlled tightly. Randomisation was balanced within centres and is unlikely to have affected our results. A corollary is that our results are generalisable to a broad range of practice styles. Use of an equally weighed composite endpoint could be judged a limitation, but we have provided insight into the distribution of events by presenting the incidence of every complication separately.

In conclusion, during mechanical ventilation with protective low tidal volumes in patients undergoing open abdominal surgery, use of a high level of positive endexpiratory pressure and recruitment manoeuvres does not reduce the incidence of postoperative pulmonary complications and more frequently results in haemodynamic instability, compared with use of low positive end-expiratory pressure without recruitment manoeuvres.

\section{Contributors}

All members of the Steering committee contributed to the design and conduct of the study. Data collection was undertaken by PROVE Network Collaborators (appendix pp 2-3). The report was written by the Writing committee and revised by the Steering committee. JMB and SNTH had complete access to all the data in the study and did data analysis, with help from MJS, MGdA, and PP. SNTH, MJS, MGdA, and PP made the final decision to submit the report for publication. SNTH, MJS, MGdA, and PP contributed equally to the study.

\section{Writing committee}

Sabrine N T Hemmes (Academic Medical Center, University of Amsterdam, Amsterdam, Netherlands); Marcelo Gama de Abreu (University Hospital Dresden, Technische Universität Dresden, Dresden, Germany); Paolo Pelosi (Università degli Studi di Genova, IRCCS San Martino IST, Genoa, Italy); Marcus J Schultz (Academic Medical Center, University of Amsterdam, Amsterdam, Netherlands).

\section{Steering committee}

Sabrine N T Hemmes (Academic Medical Center, University of Amsterdam, Amsterdam, Netherlands); Marcelo Gama de Abreu (University Hospital Dresden, Technische Universität Dresden, Dresden, Germany); Paolo Severgnini (University of Insubria, Varese, Italy); Markus W Hollmann (Academic Medical Center, University of Amsterdam, Amsterdam, Netherlands); Jan M Binnekade [study statistician] (Academic Medical Center, University of Amsterdam, Amsterdam, Netherlands); Hermann Wrigge (University of Leipzig, Germany); Jaume Canet (Hospital Universitari Germans Trias I Pujol, Barcelona, Spain); Michael Hiesmayr (Medical University, Vienna, Austria); Werner Schmid (Medical University, Vienna, Austria); Samir Jaber (Saint Eloi University Hospital, Montpellier, France); Göran Hedenstierna (University Hospital, Uppsala, Sweden); Christian Putensen (University Hospital, Bonn, Germany); Paolo Pelosi (Università degli Studi di Genova, IRCCS San Martino IST, Genoa, Italy); Marcus J Schultz (Academic Medical Center, University of Amsterdam, Amsterdam, Netherlands).

\section{Declaration of interests}

We declare that we have no competing interests.

\section{Acknowledgments}

The Academic Medical Center (Amsterdam, Netherlands) and the European Society of Anaesthesiology provided financial support. We thank all participating research nurses, nurse anaesthetists, surgeons, other doctors, and patients (appendix p 4); Brigitte Leva and Sandrine Damster (Research Team at the European Society of Anaesthesiology) for assistance; Annelou van der Veen for on-site monitoring; and Daniel I Sessler for revision of the manuscript.

\section{References}

1 Weiser TG, Regenbogen SE, Thompson KD, et al. An estimation of the global volume of surgery: a modelling strategy based on available data. Lancet 2008; 372: 139-44.

2 Smetana GW, Lawrence VA, Cornell JE, and the American College of Physicians. Preoperative pulmonary risk stratification for noncardiothoracic surgery: systematic review for the American College of Physicians. Ann Intern Med 2006; 144: 581-95.

3 Pearse RM, Moreno RP, Bauer P, et al, for the European Surgical Outcomes Study (EuSOS) group for the Trials groups of the European Society of Intensive Care Medicine and the European Society of Anaesthesiology. Mortality after surgery in Europe: a 7 day cohort study. Lancet 2012; 380: 1059-65.

4 Canet J, Gallart L, Gomar C, et al, and the ARISCAT Group. Prediction of postoperative pulmonary complications in a population-based surgical cohort. Anesthesiology 2010; 113: 1338-50.

5 Serpa Neto A, Cardoso SO, Manetta JA, et al. Association between use of lung-protective ventilation with lower tidal volumes and clinical outcomes among patients without acute respiratory distress syndrome: a meta-analysis. JAMA 2012; 308: 1651-59. 
6 Lellouche F, Dionne S, Simard S, Bussières J, Dagenais F. High tidal volumes in mechanically ventilated patients increase organ dysfunction after cardiac surgery. Anesthesiology 2012; 116: 1072-82.

7 Duggan M, Kavanagh BP. Pulmonary atelectasis: a pathogenic perioperative entity. Anesthesiology 2005; 102: 838-54.

8 Putensen C, Theuerkauf N, Zinserling J, Wrigge H, Pelosi P. Meta-analysis: ventilation strategies and outcomes of the acute respiratory distress syndrome and acute lung injury. Ann Intern Med 2009; 151: 566-76.

9 Briel M, Meade M, Mercat A, et al. Higher vs lower positive end-expiratory pressure in patients with acute lung injury and acute respiratory distress syndrome: systematic review and meta-analysis. JAMA 2010; 303: 865-73.

10 Severgnini P, Selmo G, Lanza C, et al. Protective mechanical ventilation during general anesthesia for open abdominal surgery improves postoperative pulmonary function. Anesthesiology 2013; 118: $1307-21$.

11 Futier E, Constantin JM, Paugam-Burtz C, et al, and the IMPROVE Study Group. A trial of intraoperative low-tidal-volume ventilation in abdominal surgery. N Engl J Med 2013; 369: 428-37.

12 Halter JM, Steinberg JM, Schiller HJ, et al. Positive end-expiratory pressure after a recruitment maneuver prevents both alveolar collapse and recruitment/derecruitment. Am J Respir Crit Care Med 2003; 167: 1620-26.

13 Nielsen J, Østergaard M, Kjaergaard J, et al. Lung recruitment maneuver depresses central hemodynamics in patients following cardiac surgery. Intensive Care Med 2005; 31: 1189-94.

14 Carvalho AR, Pacheco SA, de Souza Rocha PV, et al. Detection of tida recruitment/overdistension in lung-healthy mechanically ventilated patients under general anesthesia. Anesth Analg 2013; 116: 677-84.

15 Hemmes SN, Severgnini P, Jaber S, et al. Rationale and study design of PROVHILO: a worldwide multicenter randomized controlled trial on protective ventilation during general anesthesia for open abdominal surgery. Trials 2011; 12: 111.

16 Arozullah AM, Daley J, Henderson WG, Khuri SF, and the The National Veterans Administration Surgical Quality Improvement Program. Multifactorial risk index for predicting postoperative respiratory failure in men after major noncardiac surgery. Ann Surg 2000; 232: 242-53.

17 Arozullah AM, Khuri SF, Henderson WG, Daley J, and the Participants in the National Veterans Affairs Surgical Quality Improvement Program. Development and validation of a multifactorial risk index for predicting postoperative pneumonia after major noncardiac surgery. Ann Intern Med 2001; 135: 847-57.
18 Hemmes SN, Serpa Neto A, Schultz MJ. Intraoperative ventilatory strategies to prevent postoperative pulmonary complications: a meta-analysis. Curr Opin Anaesthesiol 2013; 26: 126-33.

19 Tusman G, Böhm SH, Vazquez de Anda GF, do Campo JL Lachmann B. 'Alveolar recruitment strategy' improves arterial oxygenation during general anaesthesia. Br J Anaesth 1999; 82: 8-13.

20 Tusman G, Belda JF. Treatment of anesthesia-induced lung collapse with lung recruitment maneuvers. Curr Anaesth Crit Care 2010; 21: $244-49$.

21 Brismar B, Hedenstierna G, Lundquist H, Strandberg A, Svensson L, Tokics L. Pulmonary densities during anesthesia with muscular relaxation: a proposal of atelectasis. Anesthesiology 1985; 62: 422-28.

22 Hachenberg T, Lundquist H, Tokics L, Brismar B, Hedenstierna G. Analysis of lung density by computed tomography before and during general anaesthesia. Acta Anaesthesiol Scand 1993; 37: 549-55.

23 Neumann P, Rothen HU, Berglund JE, Valtysson J, Magnusson A Hedenstierna G. Positive end-expiratory pressure prevents atelectasis during general anaesthesia even in the presence of a high inspired oxygen concentration. Acta Anaesthesiol Scand 1999; 43: 295-301.

24 Lindberg P, Gunnarsson L, Tokics L, et al. Atelectasis and lung function in the postoperative period. Acta Anaesthesiol Scand 1992; 36: $546-53$.

25 Melo MF, Eikermann M. Protect the lungs during abdominal surgery: it may change the postoperative outcome. Anesthesiology 2013; 118: 1254-57.

26 Pagel P, Farber N, Pratt P, Warltier D. Cardiovascular pharmacology. In: Miller R, Eriksson L, Fleisher L, Wiener-Kronish J, Young W, eds. Miller's anesthesia, 7th edn. Philadelphia: Elsevier, 2009: pp 595-632.

27 Reves J, Glass P, Lubarsky D, McEvoy M, Martinez-Ruiz R. Intravenous anesthetics. In: Miller R, Eriksson L, Fleisher L, Wiener-Kronish J, Young W, eds. Miller's anesthesia, 7th edn. Philadelphia: Elsevier, 2009: pp 719-68.

28 Brown D. Spinal, epidural and caudal anesthesia. In: Miller R, Eriksson L, Fleisher L, Wiener-Kronish J, Young W, eds. Miller's anesthesia, 7th edn. Philadelphia: Elsevier, 2009: pp 1611-38.

29 Luecke T, Pelosi P. Clinical review: Positive end-expiratory pressure and cardiac output. Crit Care 2005; 9: 607-21. 